\title{
Complete genome anatomy of the emerging potato pathogen Dickeya solani type strain IPO $2222^{\top}$
}

\author{
Slimane Khayi ${ }^{1}$, Pauline Blin ${ }^{1}$, Teik Min Chong ${ }^{2}$, Kok-Gan Chan $^{2}$ and Denis Faure ${ }^{1 *}$
}

\begin{abstract}
Several species of the genus Dickeya provoke soft rot and blackleg diseases on a wide range of plants and crops. Dickeya solani has been identified as the causative agent of diseases outbreaks on potato culture in Europe for the last decade. Here, we report the complete genome of the D. solani IPO 2222 ${ }^{\top}$. Using PacBio and Illumina technologies, a unique circular chromosome of 4,919,833 bp was assembled. The $\mathrm{G}+\mathrm{C}$ content reaches $56 \%$ and the genomic sequence contains 4,059 predicted proteins. The ANI values calculated for D. solani IPO $2222^{\top}$ vs. other available D. solani genomes was over $99.9 \%$ indicating a high genetic homogeneity within D. solani species.
\end{abstract}

Keywords: Short genome report, Dickeya solani, Blackleg, Soft rot, Genome, Potato

\section{Introduction}

Dickeya are pectinolytic enterobacteria that cause soft rot and blackleg diseases on a wide range of crops worldwide including potato plants (Solanum tuberosum) $[1,2]$. They are equipped with an arsenal of plant-cell wall degrading enzymes that macerate tuber and stem tissues provoking disease symptoms [3]. In the beginning of the 2000's, D. solani emerged as a novel species causing blackleg and soft rot diseases on potato in Europe and Mediterranean Basin [4]. Initially, several pectinolytic strains isolated from potatoes grown in Europe and Israel, were identified as members of the Dickeya genus, but shown to exhibit distinctive genetic and physiological traits (biovar 3). Thereafter, additional phylogenetic and biochemical analyses have brought these isolates into a distinct clade called D. solani [5-8]. The D. solani strain IPO $2222^{\mathrm{T}}$ was isolated from infected potato plants in The Netherlands in 2007 [9].

To date, 12 draft genomes of $D$. solani are available in GenBank databases. Among them, the genome of the strain IPO $2222^{\mathrm{T}}$ was sequenced using 454-pyrosequencing with a low average genome coverage $(14 \times)$. The resulting draft

\footnotetext{
* Correspondence: denis.faure@i2bc.paris-saclay.fr

${ }^{1}$ Institute for Integrative Biology of the Cell (I2BC), CNRS CEA Univ. Paris-Sud, Université Paris-Saclay, Avenue de la Terrasse, 91198 Gif-sur-Yvette cedex, France

Full list of author information is available at the end of the article
}

genome is composed of 91 contigs that were assembled in a single scaffold [9]. In this report, we combined Illumina and Pacific Biosciences technologies to provide a complete genome sequence of the strain IPO $2222^{\mathrm{T}}$. We also highlighted some phylogenetic and phenotypic key-features of the D. solani species.

\section{Organism information \\ Classification and features}

D. solani IPO $2222^{\mathrm{T}}$ belongs to the order of Enterobacteria and the class of Gammaproteobacteria. The gapAbased phylogenetic tree (Fig. 1) was congruent with the previously reported trees inferred from MLSA $[8,10]$, gathering all $D$. solani strains in a distinct clade within the Dickeya genus. The gapA housekeeping gene was chosen instead of 16S rRNA gene because the sequence analysis of gapA permit a highly resolved view of distinction between members of the Dickeya genus $[8,10]$.

D. solani IPO $2222^{\mathrm{T}}$ is a Gram negative, non-sporeforming, motile and facultative anaerobic bacterium with rod shaped cells $(0.9 \times 2.0 \mu \mathrm{m})$ (Fig. 2) [8]. The strain IPO $2222^{\mathrm{T}}$ grows in TY medium (tryptone $5 \mathrm{~g} / \mathrm{L}$, yeast extract $3 \mathrm{~g} / \mathrm{L}$ and agar $1.5 \%$ ) at $28{ }^{\circ} \mathrm{C}$ forming $1-2 \mathrm{~mm}$ colonies within $24 \mathrm{~h}$. It produces phosphatase and indole and belongs to Dickeya biovar 3 as described previously [10]. Distinctive metabolic abilities of $D$. solani species were described using BIOLOG system [11]; among them, D. solani 


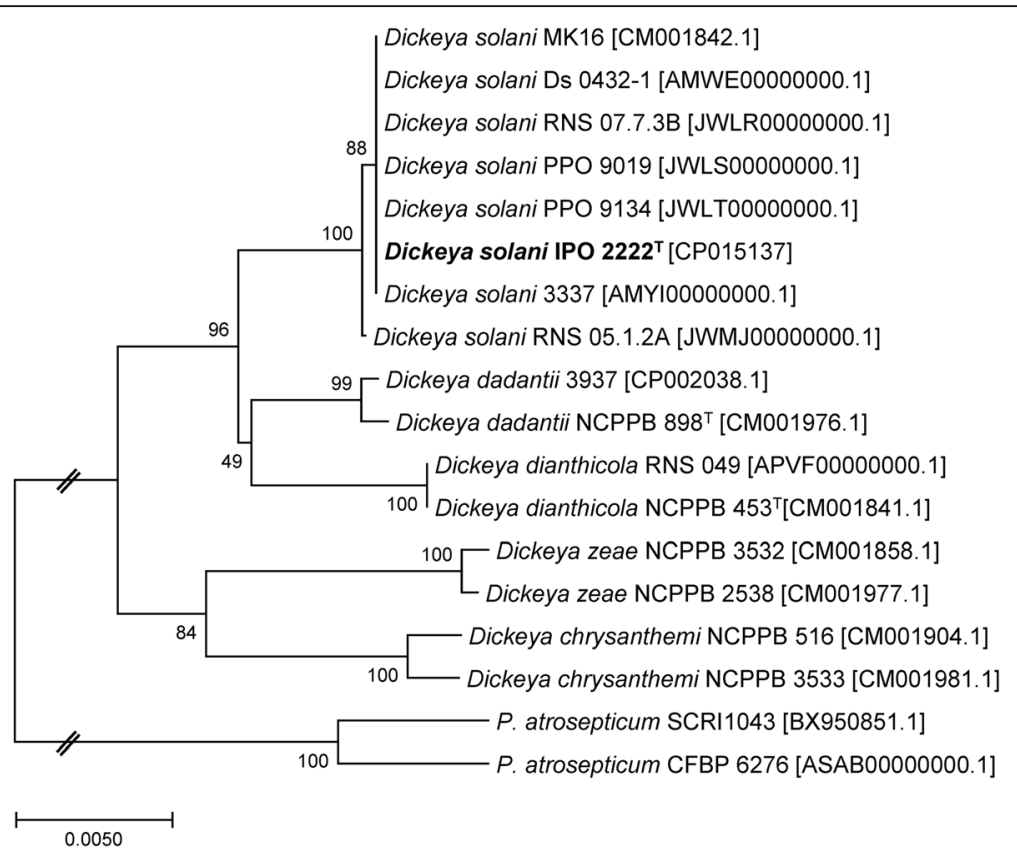

Fig. 1 Phylogenetic tree highlighting the relative position of D. solani IPO $2222^{\top}$ within other Dickeya and Pectobacterium species. The unique gapA gene was retrieved from each of the complete and draft genomes that are available in NCBI database; alignment was generated using MUSCLE [23]; the evolutionary history was inferred using the Neighbor-Joining method [24] and the evolutionary distances were computed using the Maximum Composite Likelihood method [25]. Phylogenetic analyses were conducted using MEGA7 software [26]

IPO $2222^{\mathrm{T}}$ uses urea as sole nitrogen source (Additional file 1: Figure S1). D. solani IPO $2222^{\mathrm{T}}$ was recovered form naturally infected potato plants showing blackleg and soft rot symptoms. Its aggressiveness was confirmed by infecting potato tubers and plants in greenhouse assays (Additional file 2: Figure S2). In addition, its ability to colonize the roots and stem tissues and to provoke disease symptoms has been reported using green fluorescent protein-tagged strain [12].

The strain IPO $2222^{\mathrm{T}}$ has been registered at the Belgian Co-ordinated Collections of Micro-organisms (LMG $25993^{\mathrm{T}}$ ), the National Collection of Plant Pathogenic Bacteria in UK $\left(\mathrm{NCPPB} 4479^{\mathrm{T}}\right)$, and the International Center for Microbial Resources - French collection of

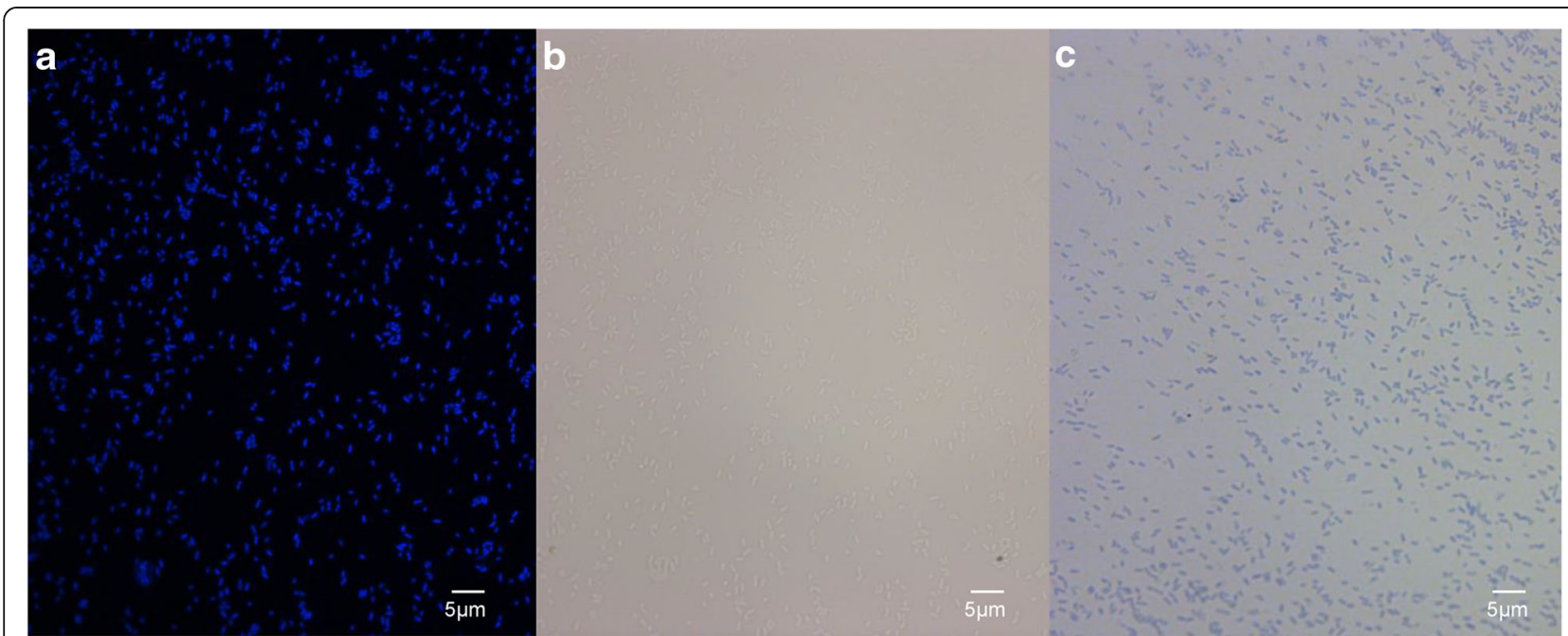

Fig. 2 Photomicrographs of D. solani IPO $2222^{\top}$ using DAPI (4,6-diamidino-2-phenylindole) staining (a), differential interference contrast (b) and blue methylene staining (c). These photomicrographs show the rod shaped forms of $D$. solani species 
plant-associated bacteria $\left(\right.$ CFBP $\left.8199^{\mathrm{T}}\right)$. MIGS of D. solani strain IPO $2222^{\mathrm{T}}$ is summarized in Table 1.

\section{Genome sequencing information Genome project history}

The genome sequence of $D$. solani strain IPO $2222^{\mathrm{T}}$ was sequenced using two technologies, PacBio RSII and Illumina NextSeq 500. This organism was selected based on the agricultural relevance as an emerging pathogen with a significant impact on the potato production and trade in Europe and around the

Table 1 Classification and general features of Dickeya solani strain IPO $2222^{\top}$ [13]

\begin{tabular}{|c|c|c|c|}
\hline$\overline{M I G S I D}$ & Property & Term & $\begin{array}{l}\text { Evidence } \\
\text { code }^{\mathrm{a}}\end{array}$ \\
\hline & Classification & Domain Bacteria & TAS [15] \\
\hline & & Phylum Proteobacteria & TAS [27] \\
\hline & & Class Gammaproteobacteria & $\begin{array}{l}\text { TAS } \\
{[28,29]}\end{array}$ \\
\hline & & Order "Enterobacteriales" & $\begin{array}{l}\text { TAS } \\
{[28,29]}\end{array}$ \\
\hline & & Family Enterobacteriaceae & TAS [30] \\
\hline & & Genus Dickeya & TAS [1] \\
\hline & & Species Dickeya solani & TAS [8] \\
\hline & & $\begin{array}{l}\text { Type strain: IPO } 2222^{\top} \\
\text { (CP015137) }\end{array}$ & \\
\hline & Gram stain & negative & TAS [8] \\
\hline & Cell shape & Rod & TAS [8] \\
\hline & Motility & Motile & IDA \\
\hline & Sporulation & Non sporulating & NAS [8] \\
\hline & Temperature range & Mesophilic & TAS [8] \\
\hline & $\begin{array}{l}\text { Optimum } \\
\text { temperature }\end{array}$ & $39^{\circ} \mathrm{C}$ & TAS [8] \\
\hline & $\mathrm{pH}$ range; Optimum & Not reported;7 & IDA \\
\hline & Carbon source & D-Arabinose, Mannitol & TAS [8] \\
\hline MIGS-6 & Habitat & Rhizosphere & TAS [8] \\
\hline MIGS-6.3 & Salinity & $0.5 \% \mathrm{NaCl}(\mathrm{w} / \mathrm{v})$ & TAS [31] \\
\hline MIGS-22 & Oxygen requirement & Facultatively anaerobic & TAS [8] \\
\hline MIGS-15 & Biotic relationship & free-living & TAS [8] \\
\hline MIGS-14 & Pathogenicity & Pathogenic & NAS [8] \\
\hline MIGS-4 & Geographic location & Netherlands & $\operatorname{TAS}[8,9]$ \\
\hline MIGS-5 & Sample collection & 2007 & $\operatorname{TAS}[8,9]$ \\
\hline MIGS-4.1 & Latitude & Not reported & NAS \\
\hline MIGS-4.2 & Longitude & Not reported & NAS \\
\hline MIGS-4.4 & Altitude & Not reported & NAS \\
\hline
\end{tabular}

${ }^{a}$ Evidence codes - IDA: Inferred from Direct Assay; TAS: Traceable Author Statement (i.e., a direct report exists in the literature); NAS: Non-traceable Author Statement (i.e., not directly observed for the living, isolated sample, but based on a generally accepted property for the species, or anecdotal evidence). These evidence codes are from the Gene Ontology project [32] world. Project information is available from Genome Online database number Gp0138842 under the Gold study number Gs0118682 at Joint Genome Institute. The complete genome sequence is also deposited in GenBank under the accession number CP015137. In Table 2, we provide a summary of the project information and its association with MIGS [13].

\section{Growth conditions and genomic DNA preparation}

D. solani IPO $2222^{\mathrm{T}}$ was routinely cultured in TY medium at $28{ }^{\circ} \mathrm{C}$. Genomic DNA extraction was performed from $5 \mathrm{~mL}$ overnight culture using a phenolchloroform purification method followed by an ethanol precipitation as described by Wilson [14]. Quantification and quality control of the DNA was completed using a NanoDrop (ND 1000) device, Qubit 2.0 fluorometer and agarose $(1.0 \%)$ gel electrophoresis.

\section{Genome sequencing and assembly}

Second generation sequencing was performed using NextSeq 500 (Illumina, CA, USA) at the I2BC platform (Gif-sur-Yvette, France). A paired-end library was constructed with an insert size of $390 \mathrm{bp}$ and sequencing was carried out using $2 \times 151$ bp paired-end read module. The de novo assembly (length fraction, 0.5 ; similarity, 0.8 ) was performed using CLC Genomics Workbench (v8.0) software (CLC Inc, Aarhus, Denmark). After quality (quality score threshold 0.05 ) and length (above 40 nucleotides) trimming of the sequences, 33 contigs $(\mathrm{N} 50=266,602 \mathrm{bp}$ ) were generated (CLC parameters: automatic determination of the word and bubble sizes with no scaffolding) with a $450 \times$ average genome coverage. The largest contig length was $617,431 \mathrm{bp}$.

Third generation sequencing was performed using PacBio RSII (Pacific Biosciences, CA, USA) at the University of

Table 2 Project information

\begin{tabular}{|c|c|c|}
\hline$\overline{M I G S I D}$ & Property & Term \\
\hline MIGS 31 & Finishing quality & Complete genome \\
\hline MIGS-28 & Libraries used & Paired-end \\
\hline MIGS 29 & Sequencing platforms & Illumina NextSeq500, PacBio \\
\hline MIGS 31.2 & Fold coverage & $450 \mathrm{X}$ \\
\hline MIGS 30 & Assemblers & CLC Genomics \\
\hline \multirow[t]{6}{*}{ MIGS 32} & Gene calling method & $\begin{array}{l}\text { NCBI Prokaryotic Genome } \\
\text { Annotation Pipeline }\end{array}$ \\
\hline & Locus Tag & $\mathrm{A} 4 \cup 42$ \\
\hline & Genbank ID & CP015137 \\
\hline & GenBank Date of Release & 16 Mai 2016 \\
\hline & GOLD ID & Gp0138842 \\
\hline & BIOPROJECT & PRJNA317288 \\
\hline \multirow[t]{2}{*}{ MIGS 13} & Source Material Identifier & IPO $2222^{\top}$ \\
\hline & Project relevance & Agricultural \\
\hline
\end{tabular}


Table 3 Genome statistics

\begin{tabular}{lll}
\hline Attribute & Value & \% of total \\
\hline Genome size (bp) & $4,919,833$ & 100.00 \\
DNA coding (bp) & $4,243,944$ & 86.33 \\
DNA G + C (bp) & $2,767,155$ & 56.24 \\
DNA scaffolds & 1 & 100.00 \\
Total genes & 4,208 & 100.00 \\
Protein coding genes & 4,104 & 97.5 \\
RNA genes & 104 & 2.5 \\
Pseudo genes & 45 & 1.06 \\
Genes in internal clusters & 1,093 & 25.97 \\
Genes with function prediction & 3,670 & 87.21 \\
Genes assigned to COGs & 3,365 & 79.97 \\
Genes with Pfam domains & 3,788 & 99.02 \\
Genes with signal peptides & 386 & 9.17 \\
Genes with transmembrane helices & 953 & 22.65 \\
CRISPR repeats & 1 & - \\
\hline
\end{tabular}

Malaya (Kuala Lumpur, Malaysia). The SMRTbell template library at the size of $20 \mathrm{kbp}$ was constructed using the commercial Template Preparation Kit (Pacific Biosciences, CA, USA) followed by sequencing using P6/C4 sequencing chemistry with sequence collection time set at $240 \mathrm{~min}$. Prior to assembly, short reads (less than $500 \mathrm{bp}$ ) were filtered off and the minimum polymerase read quality used for mapping of sub-reads from a single zero-mode waveguides was set at 0.75 . In total 146,263 reads were obtained (N50 value was 9,161 bp) and total base pair number was at 1,070,191,526 resulting in a $217 \times$ average genome coverage. Reads were assembled using RS_HGAP_Assembly software (V2.0). The cut-off length of seeding reads was set at $13,304 \mathrm{bp}$ in order to serve as a reference for the recruitment of shorter reads for preassembly. The resulted consensus accuracy based on multiple sequence alignment of the sub-reads was at $99.99 \%$.

The de novo Illumina-contigs were used to verify the RS_HGAP assembly by blasting them against the PacBio sequence. In addition, the trimmed Illumina reads were mapped (length fraction, 0.5; similarity, 0.8) against the PacBio sequence and errors (SNPs and InDels), that might be generated by homopolymers during PacBio sequencing, were searched and corrected using basic variant calling tool from CLC genomic workbench. Using these two sets of sequences, the complete genome sequence was approved and circularized.

\section{Genome annotation}

The complete genome of D. solani IPO $2222^{\mathrm{T}}$ was annotated using the NCBI prokaryotic genome annotation pipeline [15]. The protein coding gene prediction process begin by an alignment using ProSplign [16] where only complete alignments with $100 \%$ identity to a reference protein are kept for final annotation. Then the remaining frameshift or partial alignments were further analyzed by GeneMarkS+ [17]. To identify structural rRNA, the pipeline uses BLASTn search against the curated reference set. tRNAscan-SE was used to identify the tRNAs [18]. The CRISPRs are identified by using the CRISPR database [15].

\section{Genome properties}

The detailed information about Dickeya solani IPO $2222^{\mathrm{T}}$ genome is provided in Table 3 . The genome is constituted of one circular chromosome, 4,919,833 bp in size. The annotation predicted 4,208 genes including 4,059 CDSs (Table 4), 104 RNA genes (75 tRNA, 22 rRNA and 7 ncRNA genes) and 45 pseudo genes. The

Table 4 Number of genes associated with general COG functional categories

\begin{tabular}{|c|c|c|c|}
\hline Code & Value & $\%$ age $^{a}$ & Description \\
\hline J & 234 & 6.09 & Translation, ribosomal structure and biogenesis \\
\hline A & 1 & 0.03 & RNA processing and modification \\
\hline K & 305 & 7.94 & Transcription \\
\hline $\mathrm{L}$ & 112 & 2.92 & Replication, recombination and repair \\
\hline B & 0 & 0.00 & Chromatin structure and dynamics \\
\hline $\mathrm{D}$ & 42 & 1.09 & $\begin{array}{l}\text { Cell cycle control, Cell division, chromosome } \\
\text { partitioning }\end{array}$ \\
\hline V & 90 & 2.34 & Defense mechanisms \\
\hline $\mathrm{T}$ & 216 & 5.62 & Signal transduction mechanisms \\
\hline M & 245 & 6.38 & Cell wall/membrane biogenesis \\
\hline $\mathrm{N}$ & 106 & 2.76 & Cell motility \\
\hline$U$ & 82 & 2.14 & Intracellular trafficking and secretion \\
\hline $\mathrm{O}$ & 138 & 3.59 & $\begin{array}{l}\text { Posttranslational modification, protein turnover, } \\
\text { chaperones }\end{array}$ \\
\hline C & 222 & 5.78 & Energy production and conversion \\
\hline G & 324 & 8.44 & Carbohydrate transport and metabolism \\
\hline E & 438 & 11.41 & Amino acid transport and metabolism \\
\hline $\mathrm{F}$ & 96 & 2.5 & Nucleotide transport and metabolism \\
\hline $\mathrm{H}$ & 192 & 5.0 & Coenzyme transport and metabolism \\
\hline I & 130 & 3.39 & Lipid transport and metabolism \\
\hline P & 281 & 7.32 & Inorganic ion transport and metabolism \\
\hline Q & 93 & 2.42 & $\begin{array}{l}\text { Secondary metabolites biosynthesis, transport } \\
\text { and catabolism }\end{array}$ \\
\hline $\mathrm{R}$ & 282 & 7.34 & General function prediction only \\
\hline S & 175 & 4.56 & Function unknown \\
\hline- & 843 & 20.03 & Not in COGs \\
\hline${ }^{\mathrm{b}}$ Total & 4,683 & 120 & \\
\hline
\end{tabular}




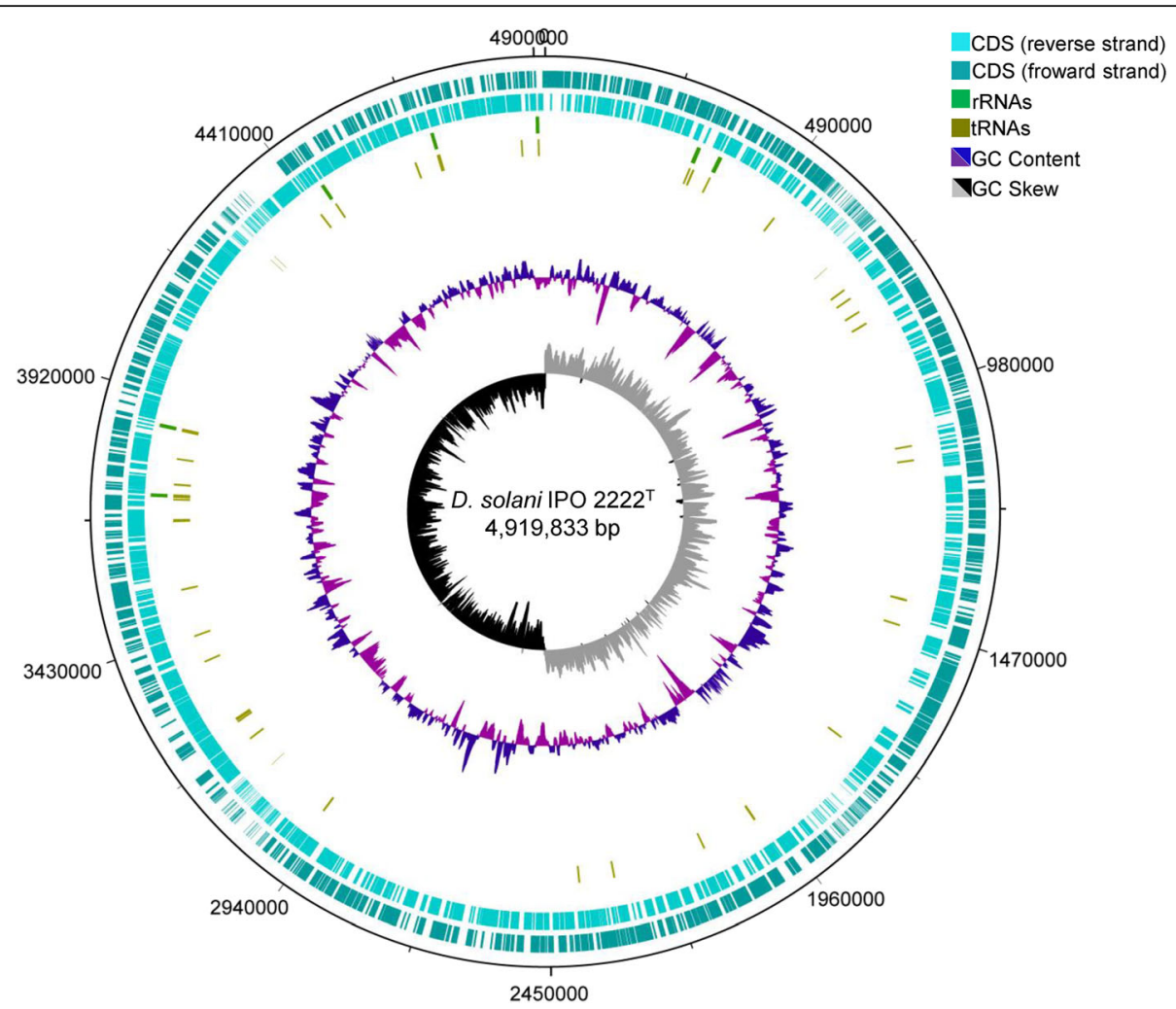

Fig. 3 Graphical circular map of D. solani IPO $2222^{\top}$ chromosome

$\mathrm{G}+\mathrm{C}$ reached $56 \%$. The graphical genome map is provided in the Fig. 3.

\section{Insights from the genome sequence}

$D$. solani species is genetically highly homogenous with 99.9\% in genomic similarity (ANI value) $[19,20]$. Between two given $D$. solani genomes, the number of variations (SNPs/InDels) is below one hundred. For example, when D. solani strain 3337 and D. solani strain IPO $2222^{\mathrm{T}}$ were compared, 49 variations were observed: 15 were located out of CDS and 34 within CDS [19]. Only a few of $D$. solani genomes (strains RNS 07.7.3B, PPO 9019 and PPO 9134) exhibited a higher number of variations $(>1000)$ because they acquired $D$. dianthicola genes by horizontal gene transfer [19]. None horizontal gene transfer from $D$. dianthicola was observed in strain IPO $2222^{\mathrm{T}}$.

Plant-cell wall degrading enzymes comprising pectinases, proteinases and cellulases, play a major role in the plant tissue maceration process [21]. Indeed, 10 pectates lyase enzymes (genes pelABCDEILXWZ) were predicted in strain IPO $2222^{\mathrm{T}}$ genome; they showed a $93.3 \%$ average nucleotide identity when compared to the orthologous genes of $D$. dadantii 3937 .

Recent comparative analyses underlined the major genetic and metabolic divergences between Dickeya solani species and the nearest clades that are $D$. dandatii (ANI 94\%) and D. dianthicola (ANI 92\%) [11, 19]. D. solani is characterized by a low content of phages elements and CRISPR system: in strain IPO $2222^{\mathrm{T}}$ genome, only one CRISPR cluster (208 bp) was identified. Using PHAST tool [22], the strain IPO $2222^{\mathrm{T}}$ harbors one questionable prophage (11 CDSs) in a 10,687 bp region. In addition, some genomic regions were shown to be specific for $D$. solani species and contain some metabolic and NRPS/PKS encoding genes [11].

\section{Conclusions}

The complete sequence of D. solani IPO $2222^{\mathrm{T}}$ is the first complete genome of a member of this species, the type strain. This work provides a substantial resource in terms of knowledge of the bacterial genetic material. It may help to understand the successful fitness of $D$. solani in invading potato fields, opening the way to new control strategies against this phytopathogen.

\section{Additional files}

Additional file 1: Figure S1. Growth curves of D. solani IPO $2222^{\top}, D$ dadantii 3937 and D. dianthicola RNS 049 in the presence of urea as a sole nitrogen source. Data were collected from duplicates. (TIFF 296 kb)

Additional file 2: Figure S2. Symptoms of D. solani IPO $2222^{\top}$ on potato plants (a) and tubers (b). (TIFF $8904 \mathrm{~kb}$ ) 


\section{Abbreviations}

CDS: Coding DNA sequence; CRISPR: Clustered regularly interspaced short palindromic repeats; MIGS: Minimum information on the genome sequence; MLSA: Multi-locus sequence analysis

\section{Acknowledgements}

We thank Peter Mergaert (I2BC, CNRS) for help in microscopy imaging. This work has benefited from the platforms and expertise of the High-throughput Sequencing Platform of I2BC (Gif-sur-Yvette, France). This work is supported by CNRS (I2BC-SE2015), Agence Nationale de la Recherche (ANR-15-CE210003), the High Impact Research Grant (UM.C/625/1/HIR/MOHE/CHAN/14/01, Grant number A-000001-50001) and the French-Malaysian exchange program awarded by French Embassy in Malaysia, the continuous support of which is acknowledged here.

\section{Authors' contributions}

SK, PB, TMC performed experiments, SK assembled and analysed genome, SK, PB, TMC, KGC, DF wrote the paper. All authors read and approved the final manuscript.

\section{Competing interests}

The authors declare that they have no competing interests.

\section{Author details}

${ }^{1}$ Institute for Integrative Biology of the Cell (I2BC), CNRS CEA Univ. Paris-Sud, Université Paris-Saclay, Avenue de la Terrasse, 91198 Gif-sur-Yvette cedex, France. ${ }^{2}$ Division of Genetics and Molecular Biology, Institute of Biological Sciences, Faculty of Science, University of Malaya, 50603 Kuala Lumpur, Malaysia.

Received: 18 May 2016 Accepted: 18 November 2016 Published online: 29 November 2016

\section{References}

1. Samson R, Legendre JB, Christen R, Fischer-Le Saux M, Achouak W, Gardan L. Transfer of Pectobacterium chrysanthemi (Burkholder et al. 1953) Brenner et al. 1973 and Brenneria paradisiaca to the genus Dickeya gen. nov. as Dickeya chrysanthemi comb. nov. and Dickeya paradisiaca comb. nov. and delineation of four novel species, Dickeya dadantii sp. nov., Dickeya dianthicola sp. nov., Dickeya dieffenbachiae sp. nov. and Dickeya zeae sp. nov. Int J Syst Evol Microbiol. 2005:55:1415-27.

2. Gardan L, Gouy C, Christen R, Samson R. Elevation of three subspecies of Pectobacterium carotovorum to species level: Pectobacterium atrosepticum sp. nov., Pectobacterium betavasculorum sp. nov. and Pectobacterium wasabiae sp. nov. Int J Syst Evol Microbiol. 2003;53:381-91.

3. Collmer A, Keen NT. The role of pectic enzymes in plant pathogenesis. Annu Rev Phytopathol. 1986;24:383-409.

4. Toth IK, van der Wolf JM, Saddler G, Lojkowska E, Hélias V, Pirhonen M, et al. Dickeya species: an emerging problem for potato production in Europe: Dickeya spp. on potato in Europe. Plant Pathol. 2011;60:385-99.

5. Tsror L, Erlich O, Lebiush S, Hazanovsky M, Zig U, Slawiak M, et al. Assessment of recent outbreaks of Dickeya sp. (syn. Erwinia chrysanthemi) slow wilt in potato crops in Israel. Eur J Plant Pathol. 2009;123:311-20.

6. Parkinson N, Stead D, Bew J, Heeney J, Tsror Lahkim L, Elphinstone J. Dickeya species relatedness and clade structure determined by comparison of recA sequences. Int J Syst Evol Microbiol. 2009;59:2388-93.

7. Laurila J, Ahola V, Lehtinen A, Joutsjoki T, Hannukkala A, Rahkonen A, et al Characterization of Dickeya strains isolated from potato and river water samples in Finland. Eur J Plant Pathol. 2008;122:213-25.

8. van der Wolf JM, Nijhuis EH, Kowalewska MJ, Saddler GS, Parkinson N, Elphinstone JG, et al. Dickeya solani sp. nov., a pectinolytic plant-pathogenic bacterium isolated from potato (Solanum tuberosum). Int J Syst Evol Microbiol. 2014:64:768-74.

9. Pritchard L, Humphris S, Baeyen S, Maes M, Vaerenbergh JV, Elphinstone J, et al. Draft Genome Sequences of Four Dickeya dianthicola and Four Dickeya solani Strains. Genome Announc. 2013;1:e00087-12.

10. Sławiak M, van Beckhoven JRCM, Speksnijder AGCL, Czajkowski R, Grabe G, van der Wolf JM. Biochemical and genetical analysis reveal a new clade of biovar 3 Dickeya spp. strains isolated from potato in Europe. Eur J Plant Pathol. 2009;125:245-61.
11. Pédron J, Mondy S, Raoul des Essarts Y, Van Gijsegem F, Faure D. Genomic and metabolic comparison with Dickeya dadantii 3937 reveals the emerging Dickeya solani potato pathogen to display distinctive metabolic activities and T5SS/T6SS-related toxin repertoire. BMC Genomics. 2014;15:283.

12. Czajkowski R, de Boer WJ, Velvis $H$, van der Wolf JM. Systemic colonization of potato plants by a soilborne, green fluorescent protein-tagged strain of Dickeya sp. biovar 3. Phytopathology. 2010;100:134-42.

13. Field D, Garrity G, Gray T, Morrison N, Selengut J, Sterk P, et al. The minimum information about a genome sequence (MIGS) specification. Nat Biotechnol. 2008;26:541-7.

14. Wilson K. Preparation of Genomic DNA from Bacteria. Curr Protoc Mol Biol John Wiley \& Sons, Inc; 2001. Available from: http://dx.doi.org/10.1002/ 0471142727.mb0204s56

15. Woese CR, Kandler O, Wheelis ML. Towards a natural system of organisms: proposal for the domains Archaea, Bacteria, and Eucarya. Proc Natl Acad Sci. 1990;87:4576-9.

16. Wheeler DL. Database resources of the National Center for Biotechnology Information. Nucleic Acids Res. 2006;34:D173-80.

17. Besemer J. GeneMarkS: a self-training method for prediction of gene starts in microbial genomes. Implications for finding sequence motifs in regulatory regions. Nucleic Acids Res. 2001;29:2607-18.

18. Lowe TM, Eddy SR. tRNAscan-SE: A program for improved detection of transfer RNA Genes in genomic sequence. Nucleic Acids Res. 1997;25:955-64.

19. Khayi S, Blin P, Pédron J, Chong T-M, Chan K-G, Moumni M, et al. Population genomics reveals additive and replacing horizontal gene transfers in the emerging pathogen Dickeya solani. BMC Genomics. 2015;16:788.

20. Khayi S, Mondy S, Beury-Cirou A, Moumni M, Helias V, Faure D. Genome Sequence of the Emerging Plant Pathogen Dickeya solani Strain RNS 08.23.3. 1A. Genome Announc. 2014;2:e01270-13.

21. Hugouvieux-Cotte-Pattat N, Condemine G, Nasser W, Reverchon S. Regulation of pectinolysis in Erwinia chrysanthemi. Annu Rev Microbiol. 1996;50:213-57.

22. Zhou Y, Liang Y, Lynch KH, Dennis JJ, Wishart DS. PHAST: a fast phage search tool. Nucleic Acids Res. 2011;39:W347-52.

23. Edgar RC. MUSCLE: multiple sequence alignment with high accuracy and high throughput. Nucleic Acids Res. 2004;32:1792-7.

24. Saitou N, Nei M. The neighbor-joining method: a new method for reconstructing phylogenetic trees. Mol Biol Evol. 1987;4(4):406-25.

25. Tamura K, Nei M, Kumar S. Prospects for inferring very large phylogenies by using the neighbor-joining method. Proc Natl Acad Sci U S A. 2004;101: 11030-5.

26. Kumar S, Stecher G, Tamura K. MEGA7: Molecular Evolutionary Genetics Analysis Version 7.0 for Bigger Datasets. Mol Biol Evol. 2016;33:1870-4.

27. Garrity GM, Bell JA, Lilburn T. Phylum XIV. Proteobacteria Phyl. nov. In: Garrity GM, Brenner DJ, Krieg NR, Staley JT, editors. Bergey's Manual of Systematic Bacteriology. Volume 2 (Part B). 2nd ed. New York: Springer; 2005. p. 1.

28. Garrity GM, Bell JA, Lilburn T. Class I. Alphaproteobacteria class. nov. Bergey's Manual Syst. Bacteriol. Springer; 2005. p. 1-574

29. Williams KP, Kelly DP. Proposal for a new class within the phylum Proteobacteria, Acidithiobacillia classis nov., with the type order Acidithiobacillales, and emended description of the class Gammaproteobacteria. Int J Syst Evol Microbiol. 2013;63:2901-6.

30. Ewing WH, Farmer III J, Brenner DJ. Proposal of Enterobacteriaceae fam. nov., nom. rev. to replace Enterobacteriaceae Rahn 1937, nom. fam. cons. (Opin. 15, Jud. Comm. 1958), which lost standing in nomenclature on 1 January 1980. Int J Syst Evol Microbiol. 1980;30:674-5.

31. Perombelon MCM, Kelman A. Ecology of the soft rot erwinias. Annu Rev Phytopathol. 1980;18:361-87.

32. Ashburner M, Ball CA, Blake JA, Botstein D, Butler H, Cherry JM, et al. Gene ontology: tool for the unification of biology. The Gene Ontology Consortium. Nat Genet. 2000:25:25-9. 